\title{
24. COMMISSION DES PARALLAXES STELLAIRES ET DES MOUVEMENTS PROPRES
}

\section{Report of Meetings, 27 and 3r August 1964}

President: A. N. Deutsch.

SECRETARY: S. Vasilevskis.

In opening, the President announced with regret the death of Dr H. L. Alden, and gave a brief biography of the deceased member.

Then the following changes in membership of the Commission were proposed and approved: (I) Alden deleted as deceased, Jenkins and Hunter on their request. (2) Added J. Delhaye and C. A. Murray.

The Organizing Committee was nominated and approved as follows: V. V. Lavdovski, J. v. B. Lourens, W. W. Morgan, C. A. Murray, K. Aa. Strand, P. van de Kamp, S. Vasilevskis.

Paloque then proposed to substitute his report on page $33^{\circ}$ of the Transactions IAU, $\mathbf{2} \mathbf{A}$ and the corresponding references by:

Prof. E. Paloque suggests that the proper motions of all the Carte du Ciel stars should be determined. Prof. R. Bouigue particularly emphasizes the importance of stars in the Selected Areas of the Toulouse zone, proper motions of which should be determined after the $\mathrm{AGK}_{3}$ is published.

The Toulouse Observatory published the fourth catalogue of positions and proper motions of reference stars in the Toulouse zone with right ascensions between IO and II hours (7a) and between 11 and 12 hours $(7 \mathrm{~b})$.

(7a) Paloque E. Ann. Obs. Toulouse, 28, 67, 1961.

(7b) Bouigue R. Ann. Obs. Toulouse, 29, r, x963.

Murray offered the following amendments to the Greenwich reports in the Transactions $I A U, 12 \mathrm{~A}$ :

Inadvertently, no reference appeared to our work on M67. The Greenwich parallax programme has been further reduced to a total of just over roo stars. We have started taking plates of stars fainter than $m_{\mathrm{pg}}=13.5$ with proper motions larger than $I^{\prime \prime}$ in Luyten's and Giclas' catalogues.

The proposed amendments were approved.

The President then invited the members of the Commission to report on their work, in accordance with the agenda given in the Draft Reports, page lviii.

\section{A. PARALLAX OBSERVATIONS OF STARS FAINTER THAN I $3 \cdot 5$ MAgNitudes}

Strand reported on the programme with the 6I-inch astrometric reflector of the U.S. Naval Observatory located at the Station near Flagstaff, Arizona. The card catalogue of nearby stars was assembled by Worley from the Lowell Observatory survey, the Luyten proper motion catalogues and data on individual stars provided by Luyten and others. From this catalogue a provisional programme of approximately 360 stars fainter than 12.5 magnitudes and north of - $10^{\circ}$ declination was made.

Since neither gratings nor rotating sectors are used, the exposure time depends on the brightness of parallax stars. Some of the selected stars between 12.5 and 13.5 magnitudes were 
rejected for lack of suitable comparison stars, still leaving a large number of stars for parallax determination. In general all stars with annual proper motions larger than $I^{\prime \prime} \cdot O$ were included. Some selection was made of stars with proper motions from $I^{\prime \prime} \cdot 0$ to $0^{\prime \prime} \cdot 5$, while stars with proper motions between $O^{\prime \prime} \cdot 5$ and $0^{\prime \prime} \cdot 3$ were included only if they appeared of particular astrophysical interest.

All the observations are made within half an hour from the meridian to avoid effects of atmospheric dispersion. In addition, whenever feasible filters are used to limit the wavelength band. Exposure time of 10 minutes is required to obtain $17^{\text {th }}$ magnitude ( $1 \cdot 5$ magnitude above the limiting magnitude), for ${ }_{103} \mathrm{aG}$ plates with a $\mathrm{GGr}_{4}$ filter, or $\mathrm{I}$ 8th magnitude without the filter. In general two or more exposures are recorded on each plate. The camera used has automatic exposure timing and plate transport, and guiding is done directly with the right ascension and declination drives by means of a photoelectric guider of the pyramid type. Since fiduciary marks are recorded on each plate for determination of position of the optical axis, parallaxes with very high precision are expected from these plates.

Franz then reported on preliminary results obtained on the internal accuracy.

A series of 10 plates of the proper motion star G I 39-39 was measured to obtain preliminary data on the accuracy of parallax plates taken with the $6 \mathrm{I}$-inch astrometric reflector of the U.S. Naval Observatory.

The plates covered a period of only slightly more than I month. A single exposure only was measured of the parallax star and of 19 reference stars evenly distributed over the entire field. All plates were reduced to a mean plate by means of plate constants.

From the reduced co-ordinates of the parallax star its proper motion was determined by least squares solutions and found to be $\mathrm{I}^{\prime \prime} \cdot 25 /$ year at $\theta=266^{\circ}$, in close agreement with the value published by Giclas $\left(\mathrm{I}^{\prime \prime} \cdot 25 /\right.$ year at $\left.\theta=253^{\circ}\right)$. From the residuals the mean error of a position derived from one exposure on a single plate was found to be $\pm 0^{\prime \prime} \cdot 04$ (m.e.).

Provided that all precautions are taken to avoid systematic errors, the astrometric reflector may be expected to yield from one pair of plates with an epoch difference of 1 year a proper motion with a mean error of about $\pm 0^{\prime \prime} \cdot 06 /$ year (m.e.).

Van de Kamp expressed hope that the observations with the $6 \mathrm{I}$-inch reflector will be made on both sides of the meridian for evening as well as for morning stars, in order to eliminate the effect of the atmospheric dispersion.

Vasilevskis reported on the parallax programme in progress at Lick, with an automatic camera on the 36 -inch refractor. Measurable images of stars down to 17 th magnitude (approximately I magnitude above the limiting magnitude) are obtained with a 30 -minute exposure and average seeing. This exposure time has been used regardless of the brightness of the parallax stars, in order to have a uniform frame of reference stars for all the parallaxes. At the outset of the programme a rotating sector was used. It was abandoned soon because its shadow could be seen even on plates taken on moonless nights and there is a danger of systematic errors caused by the difference in the background fog. In addition, it was next to impossible to select a proper sector for variables on the programme. For handling the magnitude effects a grating is being used instead. The grating produces 4 magnitude difference between the central image and the first order spectrum, and it is being used for all the parallax stars regardless of their magnitude. Instead of trying to eliminate the magnitude equation, it is being evaluated in the process of plate solutions. The same applies to the colour effect. The abundance of the reference stars on each plate even in high galactic latitudes permits an easy selection of 20 to 30 reference stars with a sufficient range of magnitudes and colours for determination of the corresponding effects. The large number of reference stars causes no particular problems if automatic measurement and electronic computing is employed. For selection each parallax 
field is photographed in blue and yellow light with the 20-inch double astrograph. The plates of both colours then are compared by blinking and approximate colours of stars are found for selection. Then the selected stars are measured with an iris photometer and thus the data for the evaluation of the magnitude and colour effects are obtained.

There are 120 stars on the present programme, most of them are white and red dwarfs, the rest being $U$ Gem variables. It is expected that within a year a number of parallaxes of the dwarfs will be obtained from at least 20 plates for each star. Since U Gem stars are expected to have small parallaxes, they will require a larger number of plates during a longer interval.

Luyten reported on his experimental parallax work with the Palomar 48-inch Schmidt telescope. The image quality is very high, and in spite of a simple way of measurement and reduction the parallax obtained is reliable. The Palomar telescope can easily reach fainter stars than is possible either with the U.S. Naval Observatory $6 \mathrm{I}$-inch reflector or the Lick 36-inch refractor.

Murray informed that $B=20$ can just be reached in 60 minutes with the Greenwich 26 -inch refractor on unfiltered baked $\mathrm{IIaO}$ plates.

fones remarked that at Herstmonceux it is being debated whether the limiting astrometric magnitude varies with the photographic speed, aperture or some other hybrid function. It would be interesting to make closely controlled experiments so that the design of future astrographs can be optimized.

After a lively discussion on the limiting astrometric magnitude and particularly on merits and disadvantages of various approaches and methods in parallax determination, Vasilevskis proposed that the Commission consider favourably and encourage all the reported approaches and methods supplementing and checking each other in the field of parallaxes of faint stars. The proposal was approved by acclamation.

The President suggested that the Vyssotsky list of red dwarfs should be observed for parallaxes.

fones mentioned that all the stars in Gliese's catalogue with parallaxes larger than $0^{\prime \prime} \cdot 05$ and north of the equator are being observed at Herstmonceux for parallaxes. One large source for this catalogue was the Vyssotsky lists, especially for the late type dwarfs.

Gliese announced that the Catalogue of stars nearer than $20 \mathrm{pc}$ (Mitt. astr. Rechen-Inst. Heidelberg, Serie A, no. 8, 1957) is nearly out of print and the possibility of a revised second edition is being considered. For this purpose all the newly determined data for stars with parallaxes larger than $0^{\prime \prime} \cdot 04$ are being collected at Heidelberg. Observers are requested to co-operate by sending directly to Heidelberg such data if their publication is not expected in the near future.

Fredrick reported that the McCormick refractor has been repaired and improved and is again working on parallax and proper motion programmes. There are two major programmes: (I) A continuation of the programme for stars on the Vyssotsky red dwarf list. (2) Proper motions of visual double stars fainter than 9 th visual magnitude on the Scott list being done by Osvalds.

A number of parallaxes should be available soon. Also 299 red dwarfs with small or no proper motion originally rejected by Vyssotsky have been included in order to give a statistical base without the effect of selection.

\section{B. NEW TABLES FOR CORRECTIONS FROM RELATIVE TO ABSOLUTE PROPER MOTIONS}

The President invited Luyten, as the author of the topic, to give a report.

Luyten: 'My purpose in bringing up this question is to try and bring some uniformity into the proper motion situation. Here I don't mean the large programmes-zone catalogues or 
clusters usually with enough material to determine all the necessary constants and corrections, but rather the case where someone determines the proper motion of a small number of special stars-say planetaries, long period variables, Cepheids, or the like. Sometimes this is done by measuring, say, a $15^{m}$ star one is after, against a half dozen of $9^{m} \mathrm{AGK}_{2}$ or $\mathrm{FK}_{3}-4$ stars, and no matter how many electronic computers one uses I take a dim view of the systematic accuracy. But the most common method is to measure the motion of the object in question relative to 8-Io comparison stars of about the same magnitude, but then we are faced with the vexing question of the correction from relative to absolute-and this at present is a jungle. I have felt that if the Commission could agree on a method and sort of give its blessing to what are now the best known (accepted) data then with this recommendation we might have a faint hope that most people would use the same tables. One of the things to watch is that we must keep things as simple as possible, otherwise no one will use them. So I have assumed that one estimates the photographic magnitudes of the comparison stars relatively to that of the object in questionfor its magnitude will eventually be determined accurately. That then we make up (bringing Parenago tables up to date) (a) a table giving the secular parallax of stars of given photographic magnitude (not fainter than I6) and galactic latitude, $(b)$ assuming a given standard solar apex we then give a table of the factors with which these secular parallaxes must be multiplied to give the mean parallactic motion in all parts of the sky, $(c)$ a third table giving the corrections for galactic rotation, $\mathrm{A}$ and $\mathrm{B}$ terms only, and even if one feels that $R$ and $V_{\odot}$ are not too well known yet, the resultant values of $\mu_{\infty}$, $\mu_{\delta}$ will probably not change greatly. These are the same as Parenago's tables.

'Finally, if one objects that soon we may have accurate values for these corrections from the galaxies programmes-I am not so sanguine as to hope we shall have anything really definitive for at least another 30 years. If we get them sooner I am afraid we shall find they are only provisionally definitive. And we need some things now. If it is done I shall be glad to publish the tables.'

Murray stated that it is important that authors should be encouraged to publish relative proper motions as measured, and state also the estimated mean parallax of the reference stars used; better still, the results for individual stars should be given, together with a statement of how they were selected, and also what criteria were adopted for the rejection of stars with apparently large proper motion.

In reducing a relative proper motion to absolute, the greatest uncertainty arises in the mean parallaxes. Changes in the adopted constants of solar motion and differential galactic rotation can easily be allowed for in subsequent discussion.

The mean parallaxes proposed by Luyten must be critically examined, in relation to previous values (e.g. Binnendijk) before being adopted.

An extension of Oort's and Binnendijk's technique of determining mean parallaxes from proper motion dispersions, to individual fields, rather than relying on mean magnitudes or number counts, would be worth investigating. In general, magnitudes are rather uncertain, and this technique would have the advantage of being fairly independent of local fluctuations in the interstellar absorption. The velocity dispersion of a random sample of stars in a limited magnitude range should be known to, say, 25 per cent. Preliminary comparisons between different methods of deriving mean parallaxes of reference stars have been made at Herstmonceux.

We have also compared the absolute proper motions of some RR Lyrae stars, derived by the statistical method with reference to faint stars, with those derived with reference to brighter stars with supposedly known proper motions. These proper motions were derived from comparing $\mathrm{AGK}_{2}$ with the Greenwich 1900 meridian catalogue, containing the astrographic reference stars. The dispersion of residuals of the bright stars after correction for proper motion 
was no smaller than the natural dispersion of the faint stars. Some of the fault may lie in the Greenwich meridian catalogue, and I hope that $\mathrm{AGK}_{3}$ will produce better results.

Dieckvoss indicated that nobody working in the field of motions of faint stars can ever hope to use the proper motions of zone work (AGK, Yale, Cape) because the errors are much larger than the effects sought, even the zero points, including the fundamental catalogue, for the mean of a number of stars. The worker depends on the mean of the motions of faint stars. There are differences of an order of magnitude in this field of research, and the difference in stellar magnitude adds to the trouble.

The President reported on his recent results from measurement of stellar proper motions with reference to galaxies. The secular parallaxes of 13.7 photographic magnitude stars deduced from 96 pairs of plates in $3^{6}$ areas relative to 80 extragalactic nebulae seem to be greater than those derived by Binnendijk, Luyten and Deutsch, whose data for high galactic latitude are in fair agreement. The difference amounts to $0^{\prime \prime} \cdot 007$ for $b=70^{\circ}$. The timeinterval of the proper motions observations, taken with the Carte du Ciel astrograph, is about 20 years (25 areas) and 40 years ( 7 areas). The results are of preliminary character and will be verified in the near future by partial reduction of the Pulkovo and the Lick observation programmes.

After some discussions on need of new tables, Luyten asked the Commission to appoint a committee for a further study of the problem and eventual preparation of the tables. Vasilevskis proposed that the Commission authorize the President and Luyten to handle the problem and to co-opt additional members as they deem it necessary; the proposal was approved.

\section{DETERMINATION OF PROPER MOTIONS OF STAR CLUSTERS}

The President outlined the problem. The great accuracy of the relative proper motions obtained for galactic clusters of about $\pm \mathrm{O}^{\prime \prime} \cdot 00 \mathrm{I}$ is lost when reducing them to absolute proper motions. The mean parallaxes of the reference stars have statistical significance. The question is more complicated in low galactic latitudes, where the mean parallaxes may fluctuate due to interstellar absorption or to the accidental grouping of stars of different classes. Galaxies are not seen in the 'zone of avoidance' and I believe that in this case we should not neglect the absolute proper motions of bright stars in fundamental catalogues.

Then the President invited members to report on their work and to discuss the problem of proper motions of star clusters.

Murray: 'In the determination of proper motions of clusters, we have used two methods for deriving mean parallaxes of reference stars in recent studies at Herstmonceux. For M67, in which cluster members and field stars are reasonably well separated, we had accurate two-colour photometry and we were able to determine photometric parallaxes of field stars with intermediate colours on the assumption that they were dwarfs. For $\omega$ Centauri, on the other hand, in which we measured nearly 5000 stars, the separation of cluster members from field stars was only statistical on account of the small proper motion of the cluster. However, it was possible to determine the dispersions of the proper motions of the field stars in 15 different colourmagnitude groups, and by assuming the transverse velocity dispersions to be known from the kinematics of nearby stars, we estimated the mean parallaxes for each group; the resulting absolute magnitudes were reasonable. In both investigations, the underlying kinematical assumptions were confirmed by the similarity between the observed velocity ellipse, and that derived from nearby stars.'

Vasilevskis pointed out that there are two separate problems of proper motion determination of open star clusters. Only relative motions are needed for segregation of the cluster membership, and with the 'ripening' of many old collections of cluster plates the results gradually 
become more reliable and valuable. On the other hand, the problem of absolute proper motions is still in a very poor shape. Open clusters are typical disk objects and their absolute motions are valuable in the studies of the galactic rotation. Moreover, the distances of individual clusters can be better estimated than those of individual faint stars and consequently the distances can be used as parameters in investigations. It is obvious that absolute proper motions derived from relative ones by tables based on assumed secular parallaxes and constants of galactic rotation cannot serve for this type of investigation, and that absolute motions are needed with reference to either a fundamental system or to galaxies. Since practically all the open clusters are within the zone of avoidance for galaxies, a direct determination of their proper motions with respect to galaxies is impossible. There are, however, some indirect approaches and they will be tried at Lick. In any case it is being planned to measure at least 20 to 30 reference stars in a small region of each cluster in the course of the Lick proper motion programme. Since all the AGK stars measurable on Lick plates will also be measured, the proper motions of the reference stars will be obtained in the $\mathrm{FK}_{4}$ system through $\mathrm{AGK}_{3}$, and possibly also with reference to galaxies by the indirect methods. Then it will be possible to derive the absolute proper motions of open clusters directly from their existing and future relative proper motions by means of the reference stars mentioned. It should be noted that it does not matter whether these reference stars are cluster members or field stars. The cluster membership, of course, has to be known from the relative motions.

Dieckvoss reported that at Bergedorf it is being planned to determine proper motion means for about 20 open clusters with reference to $\mathrm{AGK}_{3}$ and to use these few clusters for statistical purposes.

D. THE COMPILATION OF A BIBLIOGRAPHY OF AVAILABLE CATALOGUES

AND LISTS OF PROPER MOTIONS OF STARS

The President pointed out that the data on stellar proper motions are widely scattered in various publications, and sometimes it is difficult to locate the information needed. He feels therefore that a bibliography on proper motions would be helpful.

After some discussions the President volunteered to compile such a bibliography at Pulkovo with the intention to complete it by the time of the next IAU General Assembly in 1967. The Commission approved with gratitude the proposal of the President. Financial support for publication may be needed, and in such a case it will be sought at the time of the next IAU Assembly. 